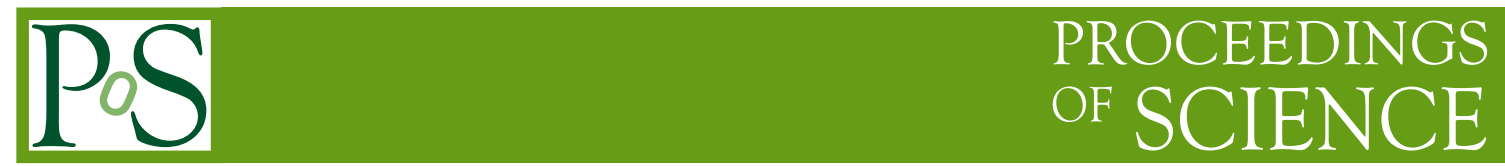

\title{
The first Be/black hole binary
}

\author{
Janusz Ziółkowski* \\ Copernicus Astronomical Center, \\ ul. Bartycka 18, 00-716 Warsaw, Poland \\ E-mail: jzdcamk.edu.p]
}

I will start with reminding (very briefly) the problem of the missing $\mathrm{Be}$ - black hole X-ray binaries. Then, I will describe the history of the discovery and the properties of the first Be - black hole X-ray binary (that was finally found). I will end with the comparison of Be X-ray binaries (including those with black holes - only one is known at present) with X-ray Novae binaries (again, including those that contain black holes).

Frontier Research in Astrophysics,

26-31 May 2014

Mondello (Palermo), Italy

${ }^{*}$ Speaker. 


\section{Introduction}

One year ago, during Frascati Workshop 2013, I was given a task of reviewing "Be/X-Ray Binaries with Black Holes in the Galaxy and in the Magellanic Clouds" (Ziółkowski, 2014). I started with the statistics indicating that the objects named in the title of my talk are either nonexisting or very elusive to detect (not a single such object was found until that time among more than 180 known Be X-ray binaries).

\section{Properties of Be/X-Ray Binaries}

Be X-ray binaries (Be XRBs) are the most numerous class among high mass X-ray binaries. We know at present 184 Be XRBs (Ziolkowski 2014 and references therein) and only about 60 other high mass X-ray binaries. These systems consist of a Be star and a compact object. Until this year, whenever the nature of the compact component was determined (in 119 systems), it was always a neutron star. The Be stars are massive, generally main sequence, stars of spectral types O8-A0 with Balmer emission lines (Negueruela 1998). The Be XRBs are rather wide systems (orbital periods in the range of $\sim 10-1180$ days). The orbits are frequently eccentric. A compact component accretes from an excretion disc (earlier known as the equatorial wind) of a Be star. In 119 systems the X-ray pulsations are observed, confirming that the compact component must be a neutron star. The pulse periods are in the range of $34 \mathrm{~ms}$ to $\sim 1400 \mathrm{~s}$.

The X-ray emission from Be XRBs (with a few exceptions) is of a distinctly transient nature with rather short (days to weeks) active phases separated by much longer (months to tens of years) quiescent intervals (a typical flaring behavior). There are two types of flares, which are classified as Type I outbursts (smaller and roughly regularly repeating) and Type II outbursts (larger and irregular. This classification was first defined by Stella et al. (1986). Type I bursts are observed in systems with highly eccentric orbits. They occur close to periastron passages of a neutron star. They are repeating at intervals $\sim P_{\text {orb }}$. Type II bursts may occur at any orbital phase. They are correlated with the disruption of the excretion disc around Be star. They repeat on time scale of the dynamical evolution of the excretion disc ( $\sim$ few years to few tens of years). This recurrence time scale is generally much longer than the orbital period (Negueruela et al. 2001).

Be XRB systems are known to contain two discs: excretion disc around Be star and accretion disc around neutron star. Both discs are temporary: excretion disc disperses and refills on time scales $\sim$ few years to few decades (dynamical evolution of the disc), while the accretion disc disperses and refills on time scales $\sim$ weeks to years (which is related either to the orbital motion of a neutron star on an eccentric orbit or to the disruption episodes of the excretion disc).

The more detailed description of the properties of Be XRBs is given, e.g. in Negueruela et al. (2001), Ziółkowski (2002), Belczyński \& Ziółkowski (2009), Ziółkowski \& Belczyński (2011), Reig (2011) and references therein.

The fact that we observe over one hundred of neutron star Be XRBs and not a single one black hole Be XRB, became known as a problem of the missing black hole Be XRBs. Trying to explain the reasons for which we do not observe black hole Be XRBs, Belczyński \& Ziółkowski (2009) carried out stellar population synthesis calculations aimed at estimating the ratio of neutron star to black hole Be XRBs, expected on the basis of the stellar evolution theory. The results of their 
calculations predict that for our Galaxy the expected ratio of Be X-ray binaries with neutron stars to the ones with black holes $F_{\mathrm{NS} / \mathrm{BH}}$ should be, most likely, equal $\sim 54$. Since we know 48 neutron star Be systems in the Galaxy, then it comes out that the expected number of black hole systems should be just one. The observed number (zero) is consistent with this prediction. In this way, the problem was solved (for galactic Be XRBs). However, the hunt for the elusive black hole Be XRB system continued. Different objects were proposed as the candidates, among them LS I $+61^{0} 303$, LS 5039 and MAXI J1836-194. None of these candidates was successful. The history of these efforts and the description of the candidate systems was given by Ziółkowski (2014).

\section{The first black hole Be XRB}

The first successful candidate was discovered as a gamma-ray source AGL J2241+4454 detected by AGILE in July 2010 (Lucarelli et al. 2010). Within two months after the discovery, Williams et al. (2010) proposed a Be star HD 215227 (known also under the name MWC 656) as an optical counterpart. The orbital period of the binary system that was probably composed of these two components (a compact object responsible for the gamma-ray emission and a Be star) was also measured and was found to be equal $60.37 \mathrm{~d}$ (based on optical photometry of a Be star). The nature of the compact component was not established at that time. Two years later, Casares et al. (2012) measured the amplitude of the radial velocities of MWC 656 and its rotational broadening using photospheric HI absorption lines. They obtained $41.7 \pm 6.8 \mathrm{~km} / \mathrm{s}$ and $346 \pm 10 \mathrm{~km} / \mathrm{s}$ respectively. Next, they analyzed Fe II lines reflecting the Keplerian rotation in the excretion disc around Be star and under some assumptions estimated the inclination of the orbit as $i=67-80^{\circ}$. Finally, they estimated the mass of the compact component to be in the range 2.7 to $5.5 \mathrm{M}_{\odot}$. This range suggested rather a black hole but a neutron star could not be excluded. Taking into account the overall low precision of this estimate, the nature of the compact component remained undecided.

After additional two years and a few more spectroscopic observations Casares et al. published the new analysis in a most recent paper in Nature (2014). This time they got two sets of radial velocities. They measured, for the first time, the accretion disc emission line He II 4686 reflecting the orbital motion of the compact component (this line was overlooked during the earlier analysis). They also improved the measurements of radial velocities of Be star by using sharp Fe II lines of the equatorial excretion disc, instead of the HeI absorption lines.

The optical spectrum of the system containing Fe II and He II emission lines is shown in Fig. 1 (after Casares et al., 2014). This picture demonstrates that both lines are indeed sharp and could be used for precise measurements of radial velocity shifts. The resulting radial velocity curves are shown in Fig. 2 (also after Casares et al., 2014). The accuracy of the measurements and the fact that the two curves are mirroring each other (they are in antiphase) leave no doubt that the shifts of the two lines in question indeed reflect the orbital motions of both components of the binary system. In this way, MWC 656 obtained the status of a bona fide double line spectroscopic binary. Using the obtained radial velocity curves, the authors determined the mass ratio (compact component to the Be star) as equal $0.41 \pm 0.07$. Then, on the basis of the improved spectral classification of the Be star (B1.5-2IIIe), they estimated the range for the probable mass of this star as $10-16 \mathrm{M}_{\odot}$. Finally, they could come to a firm conclusion that the compact component is a black hole of the mass 3.8 to $6.9 \mathrm{M}_{\odot}$. In this way the first Be/black hole binary was finally found!. 
The authors noted that the system seems to be X-ray quiescent (analysis of the archival ROSAT data leads to the upper limit $L_{\mathrm{X}}<10^{32} \mathrm{erg} / \mathrm{s}$ ). This led them to a general comment that due to lack of a solid surface and a very low mass transfer rate (leading to extremely long outburst recurrence periods), Be binaries with black hole companions might be difficult to detect by conventional Xray surveys. After completing their work, the authors obtained observing time with XMM Newton. They found that the system emits X-rays, after all (Munar-Adrover et al. 2014), but at a very low level $L_{\mathrm{X}} \approx 3.7 \times 10^{31} \mathrm{erg} / \mathrm{s}$. This level is similar to the quiescent luminosity of black hole X-Ray Novae (Garcia et al., 2001). This similarity leads us to consider the similarities and the differences between these two classes of objects i.e. Be XRBs and the X-Ray Novae.

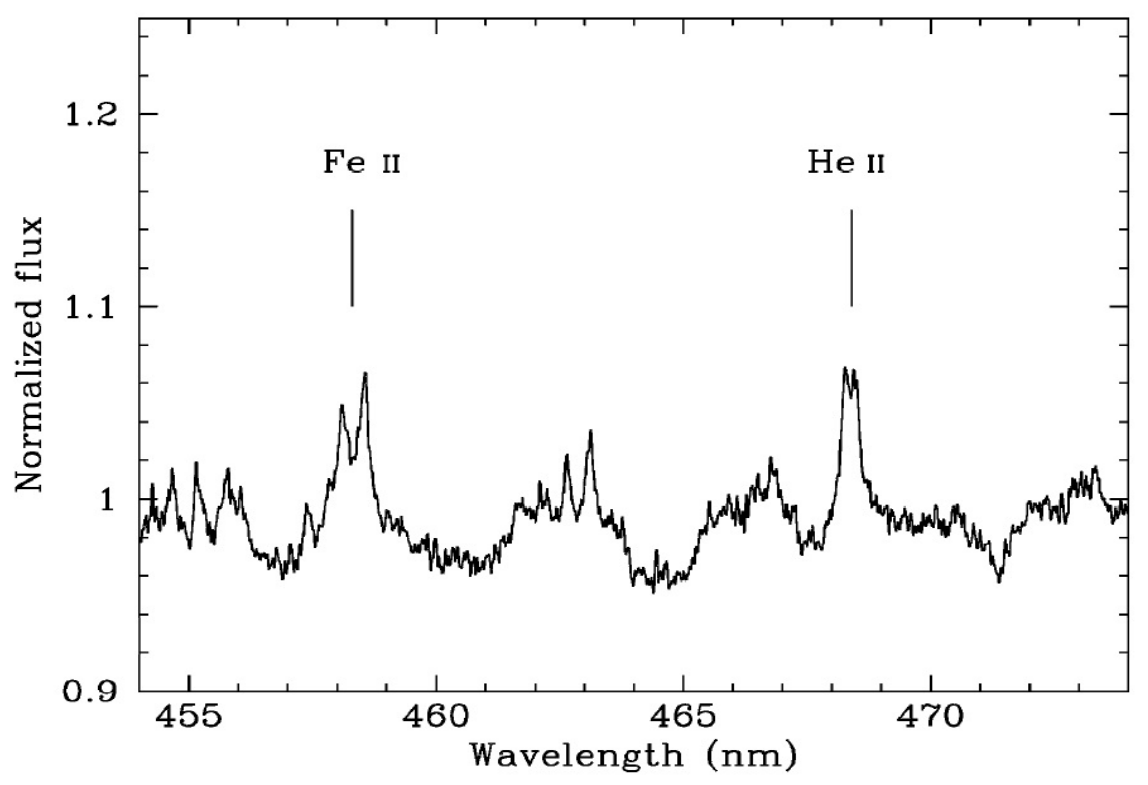

Figure 1: The optical spectrum of MWC 656 (after Casares et al. 2014).

\section{Comparison of Be/X-Ray Binaries and the X-Ray Novae}

X-Ray Novae (XRNe) are a class of low mass XRBs that are characterised by major X-ray outbursts repeating on time scale of a few months to few decades. During the outbursts, which last several weeks to several months, the luminosity might be comparable to the Eddington luminosity. Between the outbursts the luminosity (so called "quiescence luminosity") drops by six to eight orders of magnitude which indicates that the rate of the remnant accretion becomes very low. The compact component of XRNe might be a black hole or a neutron star. The quiescence luminosities of black hole systems are typically by about two orders of magnitude lower than for neutron star systems (Reynolds \& Miller 2011). This is explained as due to lack of hard surface and boundary layer in black hole systems. Table 1 lists the black hole systems for which Reynolds \& Miller 2011 reported quiescent luminosities below $10^{33} \mathrm{erg} / \mathrm{s}$.

Be XRBs, when compared with XRNe, have similar outbursts time scales and recurrence times. Also quiescence X-ray luminosities are similar. However, there are two major differences. 
Table 1: Quiescence X-Ray luminosities of black hole X-Ray Novae (after Reynolds \& Miller 2011 and references therein)

\begin{tabular}{ll}
\hline & \\
System & $L_{\mathrm{X}}^{\min }(\mathrm{erg} / \mathrm{s})$ \\
\hline & \\
\hline A0620-00 & $2.1 \times 10^{30}$ \\
GS 2000+25 & $2.4 \times 10^{30}$ \\
XTE J1650-500 & $3 \times 10^{30}$ \\
XTE J1118+480 & $3.5 \times 10^{30}$ \\
GRO J0422+32 & $7.6 \times 10^{30}$ \\
GRS 1009-45 & $<8 \times 10^{30}$ \\
GRO J1655-40 & $2.4 \times 10^{31}$ \\
4U 1543-47 & $<3 \times 10^{31}$ \\
GS 1124-683 & $4 \times 10^{31}$ \\
XTE J1819-254 & $4 \times 10^{31}$ \\
V4641 Sgr & $4.2 \times 10^{31}$ \\
H 1743-322 & $9 \times 10^{31}$ \\
XTE J1550-564 & $2 \times 10^{32}$ \\
V404 Cyg & $1.0 \times 10^{33}$ \\
& \\
\hline
\end{tabular}

Table 2: Comparison of Be XRBs and the X-Ray Novae

\begin{tabular}{lll} 
Property & Be XRBs & XRNe \\
\hline outburst mechanism & $\begin{array}{l}\text { eccentric orbit/ } \\
\text { instability of Be star disc }\end{array}$ & accretion disc instability \\
& & \\
high or low mass XRB? & HMXRB & LMXRB \\
X-ray spectrum & hard & soft \\
compact component & NS or BH & NS or BH \\
recurrence time & weeks/decades & months/decades \\
length of outburst & days/weeks & weeks/months \\
duty cycle & few $\%$ & few $\%$ \\
$L_{X}$ at quiescence $(\mathrm{erg} / \mathrm{s})$ & $10^{31}-10^{35}$ & $10^{30}-10^{33}$ \\
& &
\end{tabular}




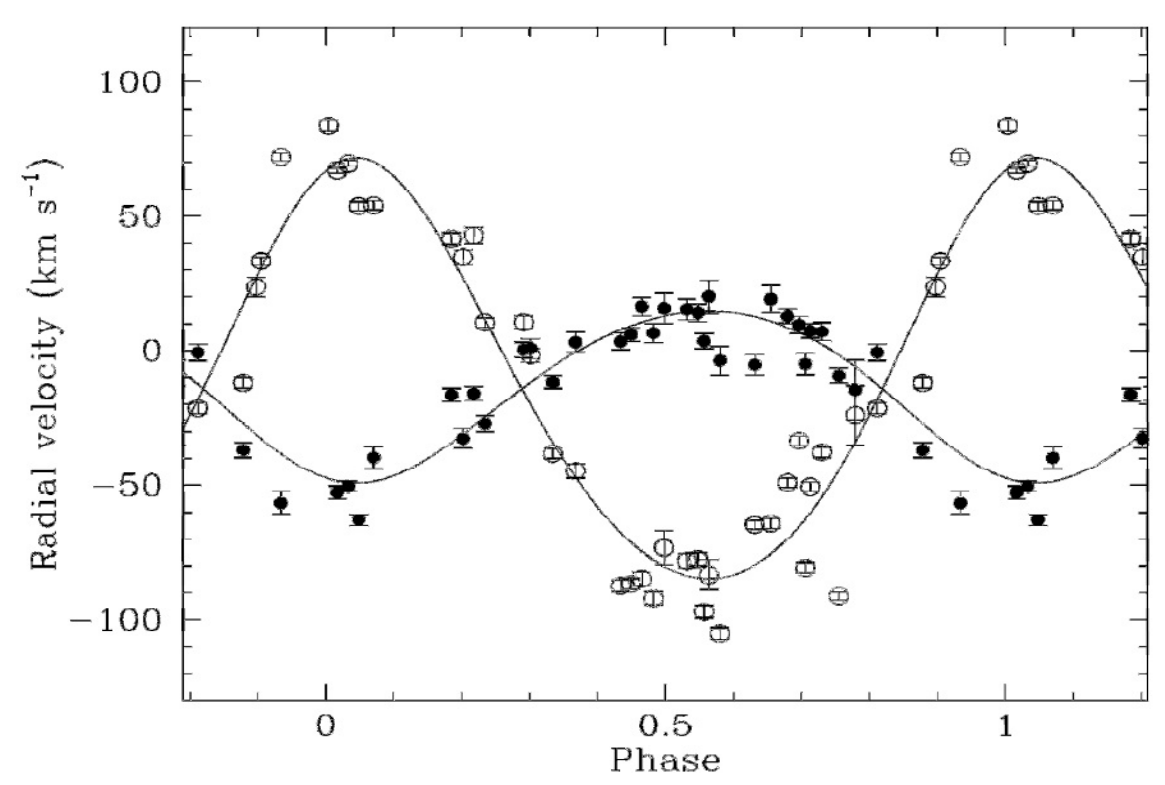

Figure 2: The radial velocity curves of the Be star and its compact companion (after Casares et al. 2014). Solid circles indicate velocities of the Be star, as obtained from Fe II 4583 line originating in the excretion disc around this star. Open circles indicate velocities of the compact component, as obtained from He II 4686 line originating in the accretion disc around this object. Error bars are 1 s.d.

First, the X-ray spectra of XRNe are soft (they originate in black holes accretion discs or in boundary layers of old (low magnetic field) neutron stars. Sometimes, the terms "soft X-ray transients" (for XRNe) and "hard X-ray transients" (for Be XRBs) are being used. The second difference is even more fundamental and concerns the mechanism of the outbursts. In XRNe it is the accretion disc instability, while in Be XRBs it is either eccentricity of the orbit (Type I outbursts) or instability of the excretion disc around Be star.

Comparison of both types of systems is given in Table 2.

\section{Conclusion}

Few years ago we predicted (Belczyński \& Ziółkowski 2009) that our Galaxy should contain just one Be XRB system containing a black hole. It seems that this system was just found.

\section{Acknowledgments}

This work was partially supported by the Polish National Science Center project 2012/04/M/ST9/00780.

\section{References}

[1] K. Belczyński \& J. Ziółkowski On the Apparent Lack of Be X-Ray Binaries with Black Holes, ApJ 707 (2009) 870 [arXiv:0907.4990] 
[2] J. Casares, I. Negueruela, M. Ribo et al. A Be-type star with a black-hole companion, (2014) [arXiv:1401.3711]

[3] J. Casares, M. Ribo, I. Ribas et al. On the binary nature of the gamma-ray sources AGL J2241+4454 (= MWC 656) and HESS J0632+057 (= MWC 148), MNRAS 421 (2012) 1103 [arXiv:1201.1726]

[4] M. Garcia, J. McClintock, R. Narayan et al. New Evidence for Black Hole Event Horizons from Chandra, ApJ 553 (2001) L47 [arXiv:astro-ph/0012452]

[5] F. Lucarelli, F. Verrecchia, E. Striani et al. AGILE detection of the new unidentified gamma-ray source AGL J2241+4454, ATel 2761 (2010)

[6] P. Munar-Adrover, J.M. Paredes, M. Ribo et al. Discovery of X-Ray Emission from the First Be/Black Hole System, ApJ 786 (2014) L11 [arXiv:1404.0901]

[7] I. Negueruela On the nature of Be/X-ray binaries, A\&A 338 (1998) 505 [arXiv:astro-ph/9807158]

[8] I. Negueruela, A.T. Okazaki, J. Fabregat et al. The Be/X-ray transient 4U 0115+63/V635 Cassiopeiae. II. Outburst mechanisms, A\&A 369 (2001) 117 [arXiv:astro-ph/0101208]

[9] P. Reig Be/X-ray binaries, Ap\&SS 332 (2011) 1 [arXiv:1101.5036]

[10] M. Reynolds \& J. Miller, J. An Anomalous Quiescent Stellar Mass Black Hole, ApJ 734 (2011) L17 [arXiv:1105.0883]

[11] L. Stella, N.E. White \& R. Rosner Intermittent stellar wind accretion and the long-term activity of Population I binary systems containing an X-ray pulsar, ApJ 308 (1986) 669

[12] S.J. Williams, D.R. Gies, R.A. Matson et al. The Be Star HD 215227: A Candidate Gamma-ray Binary, ApJ 723 (2010) L93 [arXiv:arXiv:1009.4947]

[13] J. Ziółkowski Be/X-ray binaries, MmSAI 73 (2010) 1038 [arXiv:astro-ph/0208455]

[14] J. Ziółkowski, \& K. Belczyński On the Apparent Lack of Be X-Ray Binaries with Black Holes in the Galaxy and in the Magellanic Clouds, in proceedings of Vulcano Workshop 2010: Frontier Objects in Astrophysics and Particle Physics, Conference Proceedings Italian Physical Society, Editrice Compositori, Bologna, Italy 103 (2011) 511 [arXiv:1111.2330; association of this arXiv with IAUS 275 is a mistake of ADS]

[15] J. Ziółkowski Be/X-Ray Binaries with Black Holes in the Galaxy and in the Magellanic Clouds, in proceedings of FRASCATI WORKSHOP 2013, Acta Polytechnica CTU Proceedings Czech Technical University, Prague Vol. 1, No. 1 (2014) 175 